\title{
Crystal structure of 2-Hydroxyethylamino-ethanolato-(3,5-dimethylpyrazole- $\mathrm{N}^{2}$ )-( $\boldsymbol{\mu}_{2}$-3,5-dimethylpyrazolato)-dichloride-di-copper(ii)
}

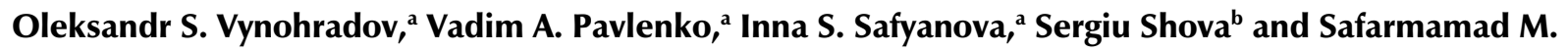 \\ Safarmamadov ${ }^{\mathrm{c} *}$ \\ aDepartment of Chemistry, Taras Shevchenko National University of Kyiv, Volodymyrska str. 64/13, 01601 Kyiv, Ukraine, b"Poni Petru" Institute \\ of Macromolecular Chemistry, Aleea Gr. Ghica, Voda 41A, 700487 lasi, Romania, and 'Department of Chemistry, Tajik National University, 17, \\ Rudaki Avenue, Dushanbe, 734025, Tajikistan \\ Correspondence email: sash65@mail.ru;_linke33967@gmail.com
}

\begin{abstract}
The title compound, $\mathrm{Cu}_{2}\left(\mathrm{C}_{5} \mathrm{H}_{8} \mathrm{~N}_{2}\right)\left(\mathrm{C}_{5} \mathrm{H}_{7} \mathrm{~N}_{2}\right)\left(\mathrm{C}_{4} \mathrm{H}_{10} \mathrm{NO}_{2}\right) \mathrm{Cl}_{2}$, is a pyrazolate aminoalcohol complex of the cyclic structure, which contains two dimethylpyrazole molecules in monodentate and bidentate-bridged coordination modes and monodeprotonated diethanolamine molecule. Both copper atoms are involved in the formation of nonplanar fivemembered chelate rings. The $\mathrm{Cu} 1$ is in a distorted tetrahedral environment formed by the pyridine nitrogen atom of the protonated dimethylpyrazole molecule, the nitrogen atom of the deprotonated bridged dimethylpyrazole, the chlorine atom and the bridged oxygen atom of the monodeprotonated diethanolamine. The $\mathrm{Cu} 2$ has an intermediate environment between trigonal-bipyramidal and square-pyramidal, formed by the nitrogen atom of the deprotonated bridged dimethylpyrazole, the chlorine atom and the nitrogen atom of aminoalcohol, and two oxygen atoms of the deprotonated and protonated $\mathrm{OH}$ groups.
\end{abstract}

\section{Chemical context}

Metal complexes of paramagnetic metal ions formed by polynucleative or polydentate ligands are of great interest as they often exhibit nontrivial magnetic behaviour (Gumienna-Kontecka et al., 2007; Suleimanov et al., 2015; Gural'skiy et al., 2012). Among polydentate and polynucleative ligands those containing both nitorgen and oxygen donor atoms are probably the most versatile and efficient chelators for the vast majority of metal ions (Pavlishchuk et al., 2010; Strotmeyer et al., 2003; Pavlishchuk et al., 2011). Amino alcohol ligands and their derivatives are one of the most widely used representatives of $\mathrm{N}, \mathrm{O}$-chelators and attract attention as a strong polydentate ligands that can form coordination compounds with transition metals (Hughes et al., 1972). Amino alcohols contain both amino and hydroxyl groups in one molecule, and therefore they are good chelating and bridging ligands. Polynuclear complexes of $3 \mathrm{~d}$ metals with amino alcohols or their deprotonated forms can show non travial properties as catalysts, materials with different magnetic properties or biologically active compounds (Reiter et al., 2006). Amino alcohol ligands are used to prepare copper (II) amino alcoholates, which, as a result of self-assembly, can form both mono- and multinuclear complexes. In bionuclear copper complexes, metal atoms can be connected by bridged oxygen atoms (alkoxy) from two different diethanolamine molecules (Tudor et al. 2003; Marin et al. 2005), or combined by a single oxygen atom from an amino alcohol and a bridged ligand molecule (Ashurov et al. 2015). There are several typical modes of binding of tridentate amino alcohol ligands to copper (II) ions and other metals such as lanthanides, yttrium, and alkaline earth metal (Breeze et al. 1994; Chen et al. 1995; Wang et al. 1995). It is a well-known fact that the copper coordination compounds can be modified with amino alcohols. For example, copper complexes with theophylline are promising objects due to their potential antitumor action and can be modified with diethanolamine by similar coordination of amino alcohols to the copper atom (Madarász et al., 2000). Studies of both tridentate and bidentate-coordinated amino alcohol ligands to the copper atom 
are being carried out (Wang 1995). Complexes of 3d-metals with a tricoordinated diethanolamine are interesting objects for synthesis and further studies (Buvaylo et al., 2009). Considering the above, we understand the importance of accumulating a theoretical information base on such coordination compounds, and therefore in this article we report the synthesis and crystal structure of a new binuclear mixed-ligand copper (II) complex containing 3,5-dimethylpyrazole and diethanolamine.

\section{Structural commentary}

The crystal structure of title compound (Fig. 3) consists of dinuclear $\mathrm{Cu}_{2}(\mathrm{Hdmpz})(\mathrm{dmpz}-\mathrm{H})(\mathrm{HDEA}) \mathrm{Cl}_{2}(\mathrm{Hdmpz}-3,5$-dimethyl-1H-pyrazole, dmpz-H - deprotonated 3,5-dimethyl-pyrazole, HDEA - monodeprotonated diethanolamine) units enclosed in two antisymmetrically oriented rows along the a axis. The unit cell consists of two unrelated structural fragments from both rows. Along the a axis within one row, each molecule is bonded to the preceding and subsequent hydrogen bonds of the same length. Along the $\mathrm{b}$ axis, the formation of molecules into dimers is due to the formation of hydrogen bonds of equal length between the bridged oxygen atom and the non deprotonated hydroxy group of the adjacent molecule. The title dinuclear pyrazolate aminoalcohol compound forms a cyclic structure. Two copper atoms with bridged oxygen atom of deprotonated diethanolamine and bridged molecule of deprotonated dimethylpyrazole form a five membered bimetallic cycle. Five-membered metallocycle has nonplanar structure. The nitrogen atoms of the bridged molecule of dimethylpyrazole are in the plane of the metallocycle while the bridged oxygen atom is out of this plane on 0.802 (1) $\AA$. The angle between the $\mathrm{Cu} 1 \mathrm{O} 1 \mathrm{Cu} 2$ and $\mathrm{Cu} 1 \mathrm{Cu} 2 \mathrm{~N} 3 \mathrm{~N} 4$ planes is $45.85(8)^{\circ}$. Geometry environment of $\mathrm{Cu} 1$ with coordination number 4 is different from $\mathrm{Cu} 2$ with coordination number 5 . The $\mathrm{Cu} 1$ is in a distorted tetrahedral environment made by the pyridine nitrogen atom of the non deprotonated dimethylpyrazole molecule, the nitrogen atom of deprotonated bridged dimethylpyrazole, the chlorine atom and the bridged oxygen atom of monodeprotonated diethanolamine. The $\mathrm{Cu} 2$ has an intermediate environment between trigonal-bipyramidal and square pyramidal, formed by the nitrogen atom of deprotonated bridged dimethylpyrazole, the chlorine atom and the nitrogen atom of aminoalcohol, and two oxygen atoms of deprotonated and non deprotonated $\mathrm{OH}$ groups. The intermetallic distance between $\mathrm{Cu} 1$ and $\mathrm{Cu} 2$ is of 3.2439 (4) $\AA$. The diethanolamine fragment is coordinated by all donor atoms to copper in a tetradentate mode and forms two similar non-planar five-membered metallocycles. It is to mentioned, that Cu2-O1 distance of 1.939 (1) $\AA$ differ significantly compared with Cu2-O2 of 2.244 (1) A.

\section{Supramolecular features}

The hydrogen bonds (Table 2) are observed between the nitrogen and the chlorine atoms (N1-H $\cdots \mathrm{Cl} 2$ and N5-H $\cdots \mathrm{C} 11)$ leading to the formation antisymmetric 1D-chains running along $a$ axis. Two 1D-chains are connected together by hydrogen bonds between hydroxyl group as donor and $\mathrm{O} 2$ atom of the adjacent molecule as acceptor of proton.

\section{Database survey}

A search of the Cambridge Structural database (CSD version 5.41 (November 2019)) for the $\mathrm{CuNH}(\mathrm{CCO})_{2}$ moiety (diethanolamine is coordinated to the copper atom) revealed one hundred and sixty-eight hits. Most similar to the title compound are the dinuclear complexes with coordinated two diethanolamine molecules, copper atoms are connected by a bridging oxygen atom and some another ligands (refcode ELESAP, Tudor et al., 2003; refcode FARKAL, Marin et al., 2005; refcode WITBAC, Madarasz et al., 2000).

\section{Synthesis and crystallization}

$1.76 \mathrm{mmol}$ diethanolamine was added by drops to the $1.15 \mathrm{mmol}$ acetonitrile solution of complex $\mathrm{Cu}_{4}\left(\mu_{2}-\mathrm{Cl}\right)_{6}\left(\mu_{4}-\mathrm{O}\right)$ $\left(\mathrm{C}_{5} \mathrm{H}_{8} \mathrm{~N}_{2}\right)_{4}$ while stirring. The mixture stirred for 2 hours with oxygen access and without heating. Amino alcohol was 
added to the brown solution and the color of mixture was changed to the green. Dark green crystals of $\mathrm{Cu}_{2}\left(\mathrm{C}_{5} \mathrm{H}_{8} \mathrm{~N}_{2}\right)$ $\left(\mathrm{C}_{5} \mathrm{H}_{7} \mathrm{~N}_{2}\right)\left(\mathrm{C}_{4} \mathrm{H}_{10} \mathrm{NO}_{2}\right) \mathrm{Cl}_{2}$ suitable for single crystal X-ray analysis were obtained by slow gas diffusion in an acetonitrile/hexane isolated system. Elemental analysis of $\mathrm{C}_{14} \mathrm{H}_{25} \mathrm{Cl}_{2} \mathrm{Cu}_{2} \mathrm{~N}_{5} \mathrm{O}_{2}$ : found $\mathrm{C} 33.96 \%, \mathrm{H} 5.267 \%$ and $\mathrm{N} 14.13 \%$ (calculated C $34.08 \%, \mathrm{H} 5.1 \%$, N $14.19 \%$ ). The yield was $55 \%$. The starting $\mathrm{Cu}_{4}\left(\mu_{2}-\mathrm{Cl}\right)_{6}\left(\mu_{4}-\mathrm{O}\right)\left(\mathrm{C}_{5} \mathrm{H}_{8} \mathrm{~N}_{2}\right)_{4}$ is a polymorphic modification of already known tetranuclear copper pyrazole-containing cluster $\mathrm{Cu}_{4} \mathrm{OCl}_{6}\left(\mathrm{C}_{5} \mathrm{H}_{8} \mathrm{~N}_{2}\right)_{4}$ and was obtained from $\mathrm{Cu}-\mathrm{CuCl}_{2} \cdot 2 \mathrm{H}_{2} \mathrm{O}-\mathrm{Hdmpz}$ system.

\section{Refinement}

Crystal data, data collection and structure refinement details are summarized in Table 1.

\section{Table 1}

Experimental details

\begin{tabular}{ll}
\hline $\begin{array}{l}\text { Crystal data } \\
\text { Chemical formula }\end{array}$ & $\mathrm{C}_{14} \mathrm{H}_{25} \mathrm{Cl}_{2} \mathrm{Cu}_{2} \mathrm{~N}_{5} \mathrm{O}_{2}$ \\
$M_{\mathrm{r}}$ & 493.37 \\
$\begin{array}{l}\text { Crystal system, space } \\
\text { group }\end{array}$ & Triclinic, $P \overline{1}$ \\
Temperature (K) & 180 \\
$a, b, c(\AA)$ & $9.0732(5), 10.7460(6), 11.5578(6)$ \\
$\alpha, \beta, \gamma\left({ }^{\circ}\right)$ & $92.373(4), 102.383(5), 112.703(5)$ \\
$V\left(\AA^{3}\right)$ & $1005.70(10)$ \\
$Z$ & 2 \\
Radiation type & $M o K \alpha$ \\
$\mu\left(\right.$ mm $\left.^{-1}\right)$ & 2.40 \\
Crystal size $(\mathrm{mm})$ & $0.4 \times 0.3 \times 0.3$
\end{tabular}

Data collection Diffractometer Absorption correction

Xcalibur, Eos

Multi-scan

CrysAlis PRO 1.171.40.53 (Rigaku Oxford Diffraction, 2019) Empirical absorption correction using spherical harmonics, implemented in SCALE3 ABSPACK scaling algorithm.

\section{$T_{\min }, T_{\max }$} $0.553,1.000$

No. of measured, $8833,4681,4108$ independent and observed $[I>2 \sigma(I)]$ reflections

$R_{\text {int }} \quad 0.018$

$(\sin \theta / \lambda)_{\max }\left(\AA^{-1}\right) \quad 0.693$

Refinement

$R\left[F^{2}>2 \sigma\left(F^{2}\right)\right], w R\left(F^{2}\right), S 0.027,0.061,1.05$

No. of reflections $\quad 4681$

No. of parameters 239

No. of restraints 3

$\mathrm{H}$-atom treatment $\quad \mathrm{H}$ atoms treated by a mixture of independent and constrained refinement $\Delta \rho_{\max }, \Delta \rho_{\min }\left(\mathrm{e} \AA^{-3}\right) \quad 0.36,-0.43$

Computer programs: CrysAlis PRO 1.171.40.53 (Rigaku OD, 2019), SHELXT (Sheldrick, 2015), SHELXL 2018/3 (Sheldrick, 2015), Olex2 1.3 (Dolomanov et al., 2009). 


\section{Table 2}

Hydrogen-bond geometry $\left(\AA,^{\circ}\right)$ for (shi_4306_)

\begin{tabular}{lllll}
\hline$D-\mathrm{H} \cdots A$ & $D-\mathrm{H}$ & $\mathrm{H} \cdots A$ & $D \cdots A$ & $D-\mathrm{H} \cdots A$ \\
\hline $\mathrm{N} 1-\mathrm{H} 1 \cdots \mathrm{Cl} 2^{\mathrm{i}}$ & $0.87(2)$ & $2.33(2)$ & $3.1201(18)$ & $152(2)$ \\
$\mathrm{N} 5-\mathrm{H} 5 \cdots \mathrm{Cl} 1^{\mathrm{ii}}$ & $0.80(2)$ & $2.84(2)$ & $3.5593(18)$ & $150(2)$ \\
$\mathrm{O} 2-\mathrm{H} 2 A \cdots \mathrm{O} 1^{\mathrm{iii}}$ & $0.85(1)$ & $1.88(1)$ & $2.7264(19)$ & $174(2)$ \\
\hline
\end{tabular}

Symmetry codes: (i) $x+1, y, z$; (ii) $x-1, y, z$; (iii) $-x,-y,-z+1$.

\section{References}

Ashurov, J. M., Ibragimov, A. B. \& Ibragimov, B. T. (2015). Polyhedron, 102, 441-446.

Breeze, S. R. \& Wang, S. (1994). Inorg. chem., 33, 5113-5121.

Buvaylo, E. A., Kokozay, V. N., Vassilyeva, O. Y., Skelton, B. W. \& Jezierska, J. (2009). Inorganica Chimica Acta, 362 (7), 2429-2434.

Chen, L., Breeze, S. R., Rousseau, R. J., Wang, S. \& Thompson, L. K. (1995). Inorg. chem., 34, 454-465.

Dolomanov, O. V., Bourhis, L. J., Gildea, R. J., Howard, J. A. K. \& Puschmann, H. (2009). J. Appl. Cryst. 42, 339-341.

Gumienna-Kontecka, E., Golenya, I. A., Dudarenko, N. M., Dobosz, A., Haukka, M., Fritsky, I. O. \& \'Swi।;atekKozVlowska, J. (2007). New J. Chem., 31, 1798-1805.

Gural'skiy, I. A., Quintero, C. M., Molnár, G., Fritsky, I. O., Salmon, L. \& Bousseksou, A. (2012). Chem. Eur. J., 18, 9946-9954.

Hughes, M. N., Waldron, B. \& Rutt, K. J. (1972). Inorganica Chimica Acta, 6, 619-622.

Madarász, J., Bombicz, P., Czugler, M. \& Pokol, G. (2000). Polyhedron, 19, 4, 457-463.

Marin, G., Tudor, V., Kravtsov, V. Ch., Schmidtmann, M., Simonov, Yu. A., Muller, A. \& Andruh, M. (2005). Cryst. Growth. Des., 5(1), 279-282.

Pavlishchuk, A. V., Kolotilov, S. V., Zeller, M., Shvets, O. V., Fritsky, I. O., Lofland, S. E., Addison, A. W. \& Hunter, A. D. (2011). Eur. J. Inorg. Chem., 31, 4826-4836.

Pavlishchuk, A. V., Kolotilov, S. V., Zeller, M., Thompson, L. K., Fritsky, I. O., Addison, A. W. \& Hunter, A. D. (2010). Eur. J. Inorg. Chem., 30, 4851-4858.

Reiter, L. G., Potaskalov, V. A., Andriiko, A. A., Kublanovsky, V. S., Pirskiy, Yu. K., Chmilenko, M. A., Lisin, V. I. \& Chmilenko, S. M. (2006). Springer. Printed in the Netherlands, pp. 333-344.

Sheldrick, G. M. (2015). Acta Cryst. A71, 3-8.

Sheldrick, G. M. (2015). Acta Cryst. C71, 3-8.

Strotmeyer, K. P., Fritsky, I. O., Ott, R., Pritzkow, H. \& Krämer, R. (2003). Supramol. Chem. 15, 529-547.

Suleimanov, I., Kraieva, O., Sánchez Costa, J., Fritsky, I. O., Molnár, G., Salmon, L. \& Bousseksou, A. (2015). J. Mater. Chem. C, 3, 5026-5032.

Tudor, V., Marin, G., Kravtsov, V., Simonov, Yu. A., Lipkowski, J., Brezeanu, M. \& Andruh, M. (2003). Inorganica Chimica Acta, 353, 35-42.

Wang, S. (1995). J. Clust. Sci., 6(4), 463-484. 
Wang, S., Pang, Z., Karen, D. L., Smith, K. D. L., Hua, Y.-S., Deslippe, C. \& Wagner, M. J. (1995). Inorg. chem., 34, 908-917.

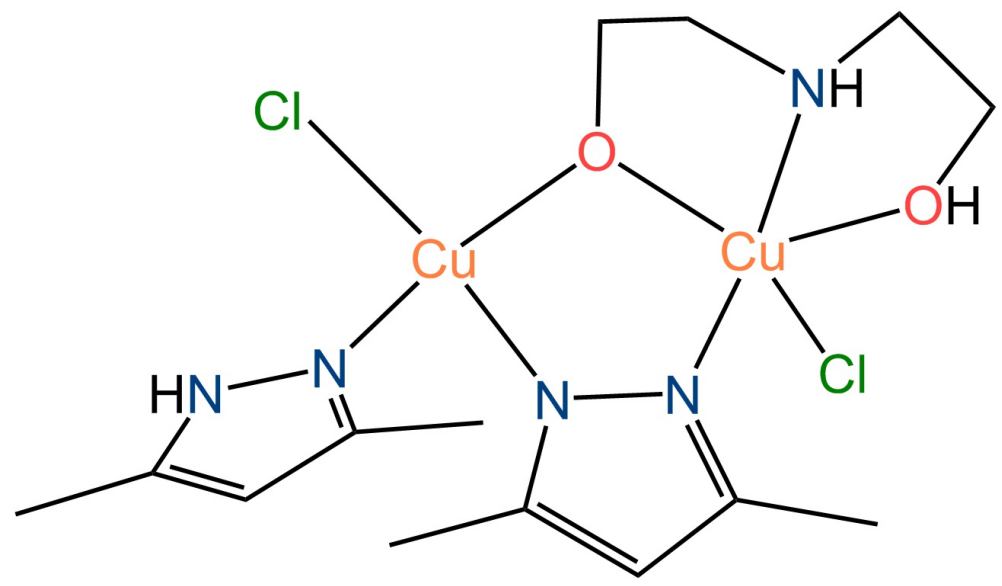

\section{Figure 1}

The structural formula of $\mathrm{Cu}_{2}\left(\mathrm{C}_{5} \mathrm{H}_{8} \mathrm{~N}_{2}\right)\left(\mathrm{C}_{5} \mathrm{H}_{7} \mathrm{~N}_{2}\right)\left(\mathrm{C}_{4} \mathrm{H}_{10} \mathrm{NO}_{2}\right) \mathrm{Cl}_{2}$

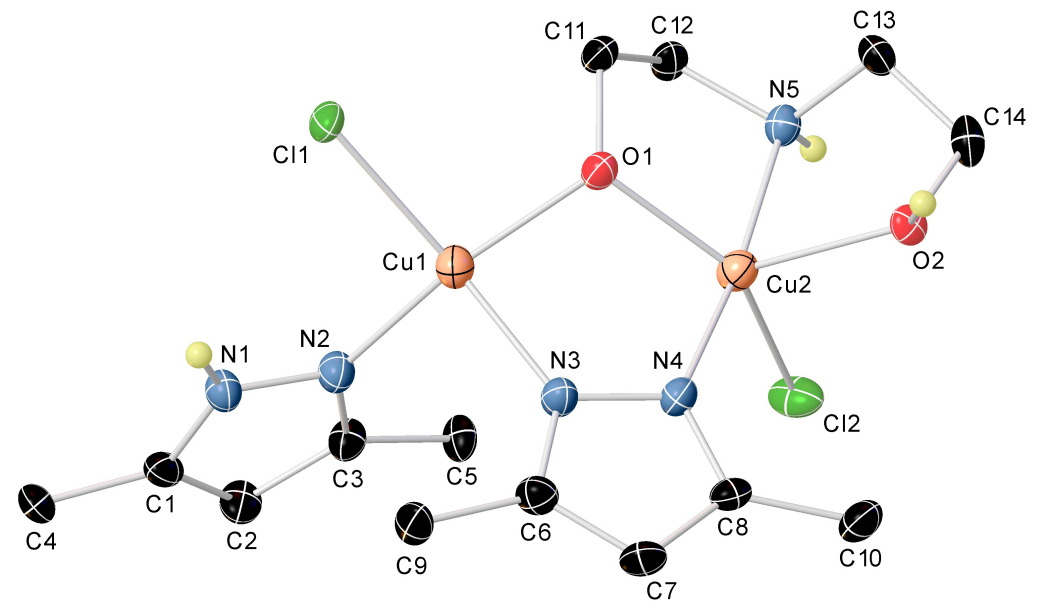

\section{Figure 2}

The molecular structure of $\mathrm{Cu}_{2}\left(\mathrm{C}_{5} \mathrm{H}_{8} \mathrm{~N}_{2}\right)\left(\mathrm{C}_{5} \mathrm{H}_{7} \mathrm{~N}_{2}\right)\left(\mathrm{C}_{4} \mathrm{H}_{10} \mathrm{NO}_{2}\right) \mathrm{Cl}_{2}$ 


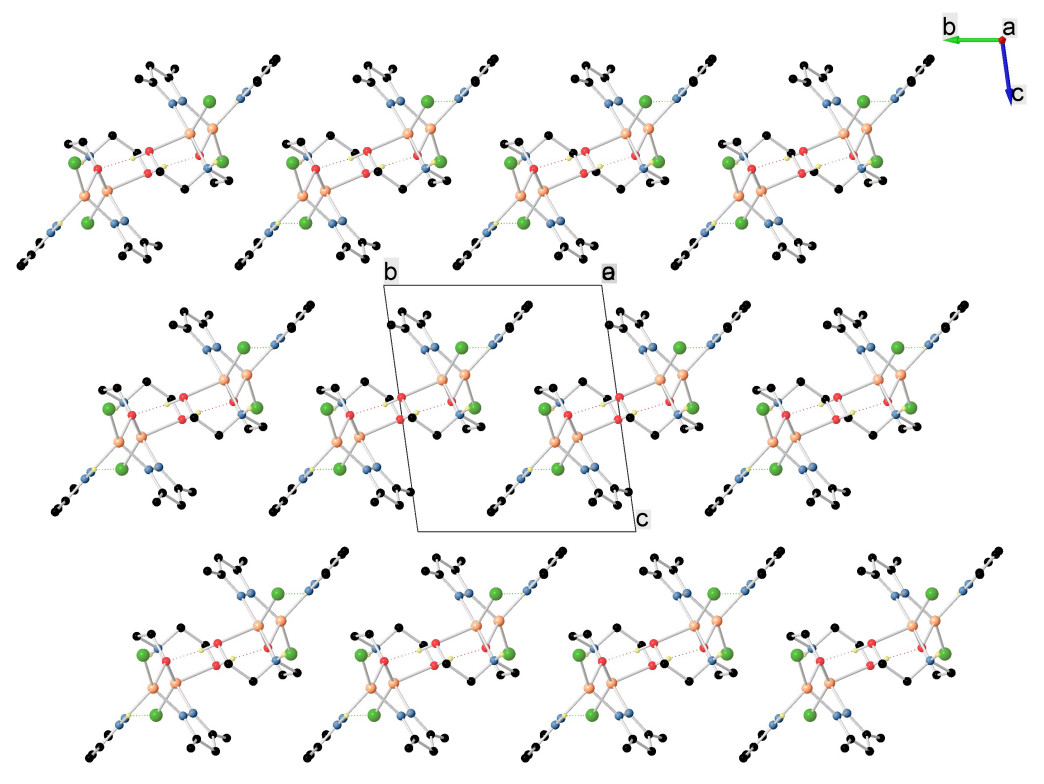

\section{Figure 3}

Crystal packing of the title compound viewed along the a axis direction

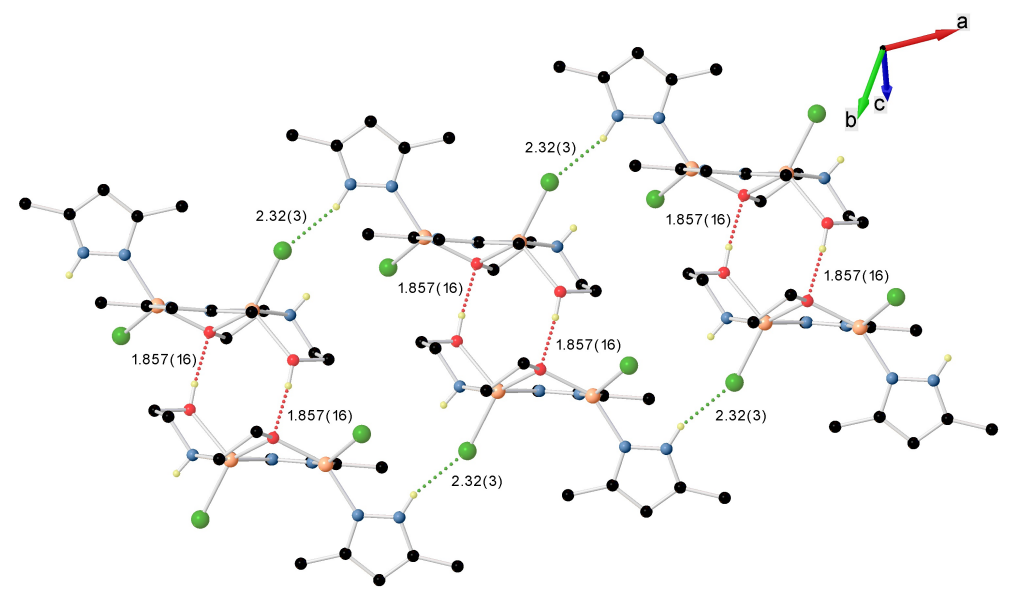

\section{Figure 4}

The illustration of hydrogen bonds in $\mathrm{Cu}_{2}\left(\mathrm{C}_{5} \mathrm{H}_{8} \mathrm{~N}_{2}\right)\left(\mathrm{C}_{5} \mathrm{H}_{7} \mathrm{~N}_{2}\right)\left(\mathrm{C}_{4} \mathrm{H}_{10} \mathrm{NO}_{2}\right) \mathrm{Cl}_{2}$ 
$\mathrm{H}$ atoms treated by a mixture of independent and constrained refinement

$w=1 /\left[\sigma^{2}\left(F_{\mathrm{o}}^{2}\right)+(0.0223 P)^{2}+0.5005 P\right]$

where $P=\left(F_{\mathrm{o}}{ }^{2}+2 F_{\mathrm{c}}{ }^{2}\right) / 3$

$$
\begin{aligned}
& (\Delta / \sigma)_{\max }=0.001 \\
& \Delta \rho_{\max }=0.36 \mathrm{e}^{-3} \\
& \Delta \rho_{\min }=-0.43 \mathrm{e} \AA^{-3}
\end{aligned}
$$

\section{Special details}

Geometry. All esds (except the esd in the dihedral angle between two 1.s. planes) are estimated using the full covariance matrix. The cell esds are taken into account individually in the estimation of esds in distances, angles and torsion angles; correlations between esds in cell parameters are only used when they are defined by crystal symmetry. An approximate (isotropic) treatment of cell esds is used

\begin{tabular}{|c|c|c|c|c|}
\hline & $x$ & $y$ & $z$ & $U_{\text {iso }} * / U_{\text {eq }}$ \\
\hline $\mathrm{C} 1$ & $0.8325(3)$ & $0.5970(2)$ & $0.87558(18)$ & $0.0213(4)$ \\
\hline $\mathrm{C} 2$ & $0.7173(3)$ & $0.6360(2)$ & $0.9068(2)$ & $0.0303(5)$ \\
\hline $\mathrm{H} 2$ & 0.737259 & 0.705862 & 0.966030 & $0.036^{*}$ \\
\hline $\mathrm{C} 3$ & $0.5648(3)$ & $0.5504(2)$ & $0.8325(2)$ & $0.0249(5)$ \\
\hline $\mathrm{C} 4$ & $1.0153(3)$ & $0.6516(2)$ & $0.9206(2)$ & $0.0299(5)$ \\
\hline $\mathrm{H} 4 \mathrm{~A}$ & 1.053223 & 0.583541 & 0.900423 & $0.045^{*}$ \\
\hline H4B & 1.045118 & 0.675213 & 1.005925 & $0.045^{*}$ \\
\hline $\mathrm{H} 4 \mathrm{C}$ & 1.065349 & 0.731079 & 0.884536 & $0.045^{*}$ \\
\hline $\mathrm{C} 5$ & $0.3987(3)$ & $0.5503(3)$ & $0.8250(3)$ & $0.0427(7)$ \\
\hline $\mathrm{H} 5 \mathrm{~A}$ & 0.360893 & 0.577420 & 0.750146 & $0.064 *$ \\
\hline $\mathrm{H} 5 \mathrm{~B}$ & 0.405616 & 0.612959 & 0.889610 & $0.064 *$ \\
\hline $\mathrm{H} 5 \mathrm{C}$ & 0.322664 & 0.460389 & 0.830295 & $0.064 *$ \\
\hline C6 & $0.3858(3)$ & $0.1471(2)$ & $0.84323(18)$ & $0.0222(5)$ \\
\hline $\mathrm{C} 7$ & $0.2607(3)$ & $0.0678(2)$ & 0.89308 (19) & $0.0237(5)$ \\
\hline $\mathrm{H} 7$ & 0.271158 & 0.021651 & 0.958574 & $0.028 *$ \\
\hline $\mathrm{C} 8$ & $0.1163(3)$ & $0.0714(2)$ & $0.82511(17)$ & $0.0189(4)$ \\
\hline C9 & $0.5663(3)$ & $0.1797(3)$ & $0.8802(2)$ & $0.0411(7)$ \\
\hline H9A & 0.604540 & 0.168147 & 0.810855 & $0.062 *$ \\
\hline H9B & 0.585441 & 0.119540 & 0.934917 & $0.062 *$ \\
\hline $\mathrm{H} 9 \mathrm{C}$ & 0.624799 & 0.272188 & 0.918419 & $0.062 *$ \\
\hline $\mathrm{C} 10$ & $-0.0560(3)$ & $0.0091(2)$ & $0.8396(2)$ & $0.0265(5)$ \\
\hline $\mathrm{H} 10 \mathrm{~A}$ & -0.073035 & 0.071365 & 0.891799 & $0.040^{*}$ \\
\hline H10B & -0.072923 & -0.073948 & 0.873356 & $0.040^{*}$ \\
\hline $\mathrm{H} 10 \mathrm{C}$ & -0.132710 & -0.010057 & 0.762932 & $0.040^{*}$ \\
\hline C11 & $0.1643(3)$ & $0.2883(2)$ & $0.41712(17)$ & $0.0192(4)$ \\
\hline H11A & 0.113125 & 0.213856 & 0.351586 & $0.023 *$ \\
\hline H11B & 0.265007 & 0.353315 & 0.401922 & $0.023^{*}$ \\
\hline $\mathrm{C} 12$ & $0.0478(3)$ & $0.3565(2)$ & $0.42571(19)$ & 0.0209 (4) \\
\hline $\mathrm{H} 12 \mathrm{~A}$ & 0.107248 & 0.443231 & 0.477042 & $0.025^{*}$ \\
\hline H12B & 0.002000 & 0.373194 & 0.347020 & $0.025^{*}$ \\
\hline $\mathrm{C} 13$ & $-0.2144(2)$ & $0.1487(2)$ & $0.39115(18)$ & $0.0210(4)$ \\
\hline H13A & -0.163870 & 0.108472 & 0.343581 & $0.025^{*}$ \\
\hline H13B & -0.289446 & 0.177492 & 0.337517 & $0.025^{*}$ \\
\hline $\mathrm{C} 14$ & $-0.3067(3)$ & $0.0466(2)$ & $0.4624(2)$ & $0.0234(5)$ \\
\hline H14A & -0.363395 & 0.085052 & 0.505581 & $0.028^{*}$ \\
\hline H14B & -0.388495 & -0.033863 & 0.409321 & $0.028 *$ \\
\hline $\mathrm{Cl1}$ & $0.57083(6)$ & $0.33924(5)$ & $0.50253(4)$ & 0.02235 \\
\hline $\mathrm{Cl} 2$ & $-0.05593(7)$ & $0.31818(6)$ & $0.74647(5)$ & $0.02968(14)$ \\
\hline
\end{tabular}
for estimating esds involving 1.s. planes.

Fractional atomic coordinates and isotropic or equivalent isotropic displacement parameters $\left(\AA^{2}\right)$ for (shi_4306_) 


\begin{tabular}{|c|c|c|c|c|}
\hline $\mathrm{Cu} 1$ & 0.41923 & $0.31469(2)$ & $0.63633(2)$ & $0.01473(7)$ \\
\hline $\mathrm{Cu} 2$ & 0.02665 & $0.20789(2)$ & $0.61602(2)$ & $0.01395(7)$ \\
\hline N1 & $0.7493(2)$ & $0.49360(18)$ & $0.78604(15)$ & $0.0175(4)$ \\
\hline H1 & $0.788(3)$ & $0.446(2)$ & $0.749(2)$ & $0.021 *$ \\
\hline $\mathrm{N} 2$ & $0.5853(2)$ & $0.46264(17)$ & $0.75898(15)$ & $0.0182(4)$ \\
\hline N3 & $0.3203(2)$ & $0.19520(17)$ & $0.74924(14)$ & $0.0172(4)$ \\
\hline N4 & $0.1545(2)$ & $0.14748(17)$ & $0.73807(14)$ & $0.0166(3)$ \\
\hline N5 & $-0.0860(2)$ & $0.26688(17)$ & $0.47523(15)$ & $0.0156(3)$ \\
\hline H5 & $-0.133(3)$ & $0.308(2)$ & $0.499(2)$ & $0.019 *$ \\
\hline $\mathrm{O} 1$ & $0.20146(16)$ & $0.23756(13)$ & $0.52738(11)$ & $0.0144(3)$ \\
\hline $\mathrm{O} 2$ & $-0.19285(18)$ & $0.01036(14)$ & $0.54515(13)$ & $0.0228(3)$ \\
\hline $\mathrm{H} 2 \mathrm{~A}$ & $-0.200(2)$ & $-0.0673(12)$ & $0.5176(19)$ & $0.034 *$ \\
\hline
\end{tabular}

Atomic displacement parameters $\left(\AA^{2}\right)$ for (shi_4306_)

\begin{tabular}{|c|c|c|c|c|c|c|}
\hline & $U^{11}$ & $U^{22}$ & $U^{33}$ & $U^{12}$ & $U^{13}$ & $U^{23}$ \\
\hline $\mathrm{C} 1$ & $0.0227(11)$ & $0.0187(11)$ & $0.0162(10)$ & $0.0030(8)$ & $0.0029(8)$ & $0.0002(8)$ \\
\hline $\mathrm{C} 2$ & $0.0311(13)$ & $0.0258(12)$ & $0.0283(12)$ & $0.0063(10)$ & $0.0088(10)$ & $-0.0104(10)$ \\
\hline $\mathrm{C} 3$ & $0.0250(12)$ & 0.0215 & $0.0293(12)$ & $0.0088(9)$ & $0.0111(9)$ & $-0.0017(9)$ \\
\hline $\mathrm{C} 4$ & $0.0221(12)$ & $0.0329(13)$ & $0.0233(12)$ & $0.0027(10)$ & $-0.0004(9)$ & $-0.0024(10)$ \\
\hline $\mathrm{C} 5$ & $0.0312(15)$ & $0.0431(16)$ & $0.0576(18)$ & $0.0179(12)$ & $0.0162(13)$ & $-0.0080(14)$ \\
\hline C6 & $0.0232(11)$ & $0.0251(12)$ & $0.0208(11)$ & $0.0128(9)$ & $0.0044(8)$ & $0.0053(9)$ \\
\hline $\mathrm{C} 7$ & $0.0303(12)$ & $0.0264(12)$ & $0.0174(10)$ & $0.0135(10)$ & $0.0071(9)$ & $0.0093(9)$ \\
\hline $\mathrm{C} 8$ & $0.0247(11)$ & $0.0188(10)$ & $0.0149(10)$ & $0.0090(9)$ & $0.0076(8)$ & $0.0027(8)$ \\
\hline C9 & $0.0271(14)$ & 0.0578 (19) & $0.0427(15)$ & $0.0214(13)$ & $0.0059(11)$ & $0.0249(13)$ \\
\hline $\mathrm{C} 10$ & $0.0276(12)$ & $0.0299(12)$ & $0.0251(11)$ & $0.0108(10)$ & $0.0132(9)$ & $0.0106(9)$ \\
\hline $\mathrm{C} 11$ & $0.0163(10)$ & $0.0270(11)$ & $0.0155(10)$ & $0.0085(9)$ & $0.0058(8)$ & $0.0080(8)$ \\
\hline $\mathrm{C} 12$ & $0.0192(11)$ & $0.0214(11)$ & $0.0231(11)$ & $0.0078(8)$ & $0.0067(8)$ & $0.0115(9)$ \\
\hline C13 & $0.0162(10)$ & 0.0233 (11) & $0.0206(10)$ & $0.0076(8)$ & $0.0003(8)$ & -0.0015 (9) \\
\hline $\mathrm{C} 14$ & $0.0148(10)$ & $0.0187(11)$ & $0.0344(12)$ & $0.0053(8)$ & $0.0054(9)$ & $-0.0027(9)$ \\
\hline $\mathrm{Cl1}$ & $0.0171(3)$ & $0.0277(3)$ & $0.0228(3)$ & $0.0076(2)$ & $0.00912(19)$ & $0.0017(2)$ \\
\hline $\mathrm{Cl} 2$ & $0.0361(3)$ & $0.0433(3)$ & $0.0217(3)$ & $0.0284(3)$ & $0.0089(2)$ & $-0.0001(2)$ \\
\hline $\mathrm{Cu} 1$ & $0.01202(13)$ & $0.01529(13)$ & $0.01474(12)$ & $0.00332(9)$ & $0.00345(9)$ & $0.00108(9)$ \\
\hline $\mathrm{Cu} 2$ & $0.01386(13)$ & $0.01565(13)$ & $0.01402(12)$ & $0.00676(10)$ & $0.00508(9)$ & $0.00341(9)$ \\
\hline N1 & $0.0144(9)$ & $0.0178(9)$ & $0.0193(9)$ & $0.0061(7)$ & $0.0034(7)$ & $-0.0003(7)$ \\
\hline N2 & $0.0153(9)$ & $0.0193(9)$ & $0.0192(9)$ & $0.0059(7)$ & $0.0052(7)$ & $0.0006(7)$ \\
\hline N3 & $0.0149(9)$ & $0.0198(9)$ & $0.0177(8)$ & $0.0073(7)$ & $0.0047(7)$ & $0.0048(7)$ \\
\hline N4 & $0.0160(9)$ & $0.0173(9)$ & $0.0175(8)$ & $0.0063(7)$ & $0.0069(7)$ & $0.0041(7)$ \\
\hline N5 & $0.0144(9)$ & $0.0148(9)$ & $0.0192(9)$ & $0.0068(7)$ & $0.0060(7)$ & $0.0014(7)$ \\
\hline $\mathrm{O} 1$ & $0.0129(7)$ & $0.0162(7)$ & $0.0143(7)$ & $0.0053(5)$ & $0.0046(5)$ & $0.0047(5)$ \\
\hline $\mathrm{O} 2$ & $0.0245(8)$ & $0.0128(7)$ & $0.0268(8)$ & $0.0043(6)$ & $0.0040(6)$ & $0.0010(6)$ \\
\hline
\end{tabular}

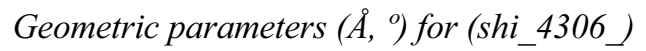

\begin{tabular}{llll}
\hline $\mathrm{C} 1-\mathrm{C} 2$ & $1.372(3)$ & $\mathrm{C} 11-\mathrm{H} 11 \mathrm{~B}$ & 0.9700 \\
$\mathrm{C} 1-\mathrm{C} 4$ & $1.490(3)$ & $\mathrm{C} 11-\mathrm{C} 12$ & $1.517(3)$ \\
$\mathrm{C} 1-\mathrm{N} 1$ & $1.341(3)$ & $\mathrm{C} 11-\mathrm{O} 1$ & $1.432(2)$ \\
$\mathrm{C} 2-\mathrm{H} 2$ & 0.9300 & $\mathrm{C} 12-\mathrm{H} 12 \mathrm{~A}$ & 0.9700 \\
$\mathrm{C} 2-\mathrm{C} 3$ & $1.393(3)$ & $\mathrm{C} 12-\mathrm{H} 12 \mathrm{~B}$ & 0.9700 \\
$\mathrm{C} 3-\mathrm{C} 5$ & $1.490(3)$ & $\mathrm{C} 12-\mathrm{N} 5$ & $1.473(3)$ \\
$\mathrm{C} 3-\mathrm{N} 2$ & $1.335(3)$ & $\mathrm{C} 13-\mathrm{H} 13 \mathrm{~A}$ & 0.9700
\end{tabular}




\begin{tabular}{|c|c|c|c|}
\hline $\mathrm{C} 4-\mathrm{H} 4 \mathrm{~A}$ & 0.9600 & $\mathrm{C} 13-\mathrm{H} 13 \mathrm{~B}$ & 0.9700 \\
\hline $\mathrm{C} 4-\mathrm{H} 4 \mathrm{~B}$ & 0.9600 & $\mathrm{C} 13-\mathrm{C} 14$ & $1.499(3)$ \\
\hline $\mathrm{C} 4-\mathrm{H} 4 \mathrm{C}$ & 0.9600 & $\mathrm{C} 13-\mathrm{N} 5$ & $1.478(2)$ \\
\hline $\mathrm{C} 5-\mathrm{H} 5 \mathrm{~A}$ & 0.9600 & $\mathrm{C} 14-\mathrm{H} 14 \mathrm{~A}$ & 0.9700 \\
\hline $\mathrm{C} 5-\mathrm{H} 5 \mathrm{~B}$ & 0.9600 & $\mathrm{C} 14-\mathrm{H} 14 \mathrm{~B}$ & 0.9700 \\
\hline $\mathrm{C} 5-\mathrm{H} 5 \mathrm{C}$ & 0.9600 & $\mathrm{C} 14-\mathrm{O} 2$ & $1.432(3)$ \\
\hline $\mathrm{C} 6-\mathrm{C} 7$ & $1.386(3)$ & $\mathrm{Cl1}-\mathrm{Cu} 1$ & $2.2403(6)$ \\
\hline $\mathrm{C} 6-\mathrm{C} 9$ & $1.494(3)$ & $\mathrm{Cl} 2-\mathrm{Cu} 2$ & $2.2937(6)$ \\
\hline $\mathrm{C} 6-\mathrm{N} 3$ & $1.344(3)$ & $\mathrm{Cu} 1-\mathrm{N} 2$ & $1.9635(16)$ \\
\hline $\mathrm{C} 7-\mathrm{H} 7$ & 0.9300 & $\mathrm{Cu} 1-\mathrm{N} 3$ & $1.9770(17)$ \\
\hline $\mathrm{C} 7-\mathrm{C} 8$ & $1.391(3)$ & $\mathrm{Cu} 1-\mathrm{O} 1$ & $1.9388(13)$ \\
\hline $\mathrm{C} 8-\mathrm{C} 10$ & $1.494(3)$ & $\mathrm{Cu} 2-\mathrm{N} 4$ & $1.9268(17)$ \\
\hline $\mathrm{C} 8-\mathrm{N} 4$ & $1.341(3)$ & $\mathrm{Cu} 2-\mathrm{N} 5$ & $1.9916(17)$ \\
\hline C9- $\mathrm{H} 9 \mathrm{~A}$ & 0.9600 & $\mathrm{Cu} 2-\mathrm{O} 1$ & $2.0001(13)$ \\
\hline C9-H9B & 0.9600 & $\mathrm{Cu} 2-\mathrm{O} 2$ & $2.2441(14)$ \\
\hline $\mathrm{C} 9-\mathrm{H} 9 \mathrm{C}$ & 0.9600 & $\mathrm{~N} 1-\mathrm{H} 1$ & $0.87(2)$ \\
\hline $\mathrm{C} 10-\mathrm{H} 10 \mathrm{~A}$ & 0.9600 & $\mathrm{~N} 1-\mathrm{N} 2$ & $1.353(2)$ \\
\hline $\mathrm{C} 10-\mathrm{H} 10 \mathrm{~B}$ & 0.9600 & $\mathrm{~N} 3-\mathrm{N} 4$ & $1.363(2)$ \\
\hline $\mathrm{C} 10-\mathrm{H} 10 \mathrm{C}$ & 0.9600 & $\mathrm{~N} 5-\mathrm{H} 5$ & $0.80(2)$ \\
\hline $\mathrm{C} 11-\mathrm{H} 11 \mathrm{~A}$ & 0.9700 & $\mathrm{O} 2-\mathrm{H} 2 \mathrm{~A}$ & $0.853(9)$ \\
\hline $\mathrm{C} 2-\mathrm{C} 1-\mathrm{C} 4$ & $131.7(2)$ & $\mathrm{N} 5-\mathrm{C} 12-\mathrm{H} 12 \mathrm{~B}$ & 109.9 \\
\hline $\mathrm{N} 1-\mathrm{C} 1-\mathrm{C} 2$ & $106.26(19)$ & $\mathrm{H} 13 \mathrm{~A}-\mathrm{C} 13-\mathrm{H} 13 \mathrm{~B}$ & 108.4 \\
\hline $\mathrm{N} 1-\mathrm{C} 1-\mathrm{C} 4$ & $122.0(2)$ & $\mathrm{C} 14-\mathrm{C} 13-\mathrm{H} 13 \mathrm{~A}$ & 110.0 \\
\hline $\mathrm{C} 1-\mathrm{C} 2-\mathrm{H} 2$ & 126.7 & $\mathrm{C} 14-\mathrm{C} 13-\mathrm{H} 13 \mathrm{~B}$ & 110.0 \\
\hline $\mathrm{C} 1-\mathrm{C} 2-\mathrm{C} 3$ & $106.60(19)$ & $\mathrm{N} 5-\mathrm{C} 13-\mathrm{H} 13 \mathrm{~A}$ & 110.0 \\
\hline $\mathrm{C} 3-\mathrm{C} 2-\mathrm{H} 2$ & 126.7 & N5- C13-H13B & 110.0 \\
\hline $\mathrm{C} 2-\mathrm{C} 3-\mathrm{C} 5$ & $129.4(2)$ & $\mathrm{N} 5-\mathrm{C} 13-\mathrm{C} 14$ & $108.28(16)$ \\
\hline $\mathrm{N} 2-\mathrm{C} 3-\mathrm{C} 2$ & $109.4(2)$ & $\mathrm{C} 13-\mathrm{C} 14-\mathrm{H} 14 \mathrm{~A}$ & 109.8 \\
\hline $\mathrm{N} 2-\mathrm{C} 3-\mathrm{C} 5$ & $121.1(2)$ & $\mathrm{C} 13-\mathrm{C} 14-\mathrm{H} 14 \mathrm{~B}$ & 109.8 \\
\hline $\mathrm{C} 1-\mathrm{C} 4-\mathrm{H} 4 \mathrm{~A}$ & 109.5 & $\mathrm{H} 14 \mathrm{~A}-\mathrm{C} 14-\mathrm{H} 14 \mathrm{~B}$ & 108.3 \\
\hline $\mathrm{C} 1-\mathrm{C} 4-\mathrm{H} 4 \mathrm{~B}$ & 109.5 & $\mathrm{O} 2-\mathrm{C} 14-\mathrm{C} 13$ & $109.25(16)$ \\
\hline $\mathrm{C} 1-\mathrm{C} 4-\mathrm{H} 4 \mathrm{C}$ & 109.5 & $\mathrm{O} 2-\mathrm{C} 14-\mathrm{H} 14 \mathrm{~A}$ & 109.8 \\
\hline $\mathrm{H} 4 \mathrm{~A}-\mathrm{C} 4-\mathrm{H} 4 \mathrm{~B}$ & 109.5 & $\mathrm{O} 2-\mathrm{C} 14-\mathrm{H} 14 \mathrm{~B}$ & 109.8 \\
\hline $\mathrm{H} 4 \mathrm{~A}-\mathrm{C} 4-\mathrm{H} 4 \mathrm{C}$ & 109.5 & $\mathrm{~N} 2-\mathrm{Cu} 1-\mathrm{Cl} 1$ & $96.92(5)$ \\
\hline $\mathrm{H} 4 \mathrm{~B}-\mathrm{C} 4-\mathrm{H} 4 \mathrm{C}$ & 109.5 & $\mathrm{~N} 2-\mathrm{Cu} 1-\mathrm{N} 3$ & $96.00(7)$ \\
\hline $\mathrm{C} 3-\mathrm{C} 5-\mathrm{H} 5 \mathrm{~A}$ & 109.5 & $\mathrm{~N} 3-\mathrm{Cu} 1-\mathrm{Cl} 1$ & $144.30(5)$ \\
\hline $\mathrm{C} 3-\mathrm{C} 5-\mathrm{H} 5 \mathrm{~B}$ & 109.5 & $\mathrm{O} 1-\mathrm{Cu} 1-\mathrm{Cl} 1$ & $98.89(4)$ \\
\hline $\mathrm{C} 3-\mathrm{C} 5-\mathrm{H} 5 \mathrm{C}$ & 109.5 & $\mathrm{O} 1-\mathrm{Cu} 1-\mathrm{N} 2$ & $148.51(7)$ \\
\hline $\mathrm{H} 5 \mathrm{~A}-\mathrm{C} 5-\mathrm{H} 5 \mathrm{~B}$ & 109.5 & $\mathrm{O} 1-\mathrm{Cu} 1-\mathrm{N} 3$ & $86.76(6)$ \\
\hline $\mathrm{H} 5 \mathrm{~A}-\mathrm{C} 5-\mathrm{H} 5 \mathrm{C}$ & 109.5 & $\mathrm{~N} 4-\mathrm{Cu} 2-\mathrm{Cl} 2$ & $95.29(5)$ \\
\hline $\mathrm{H} 5 \mathrm{~B}-\mathrm{C} 5-\mathrm{H} 5 \mathrm{C}$ & 109.5 & $\mathrm{~N} 4-\mathrm{Cu} 2-\mathrm{N} 5$ & $171.92(7)$ \\
\hline $\mathrm{C} 7-\mathrm{C} 6-\mathrm{C} 9$ & $129.3(2)$ & $\mathrm{N} 4-\mathrm{Cu} 2-\mathrm{O} 1$ & $87.45(6)$ \\
\hline $\mathrm{N} 3-\mathrm{C} 6-\mathrm{C} 7$ & $108.86(19)$ & $\mathrm{N} 4-\mathrm{Cu} 2-\mathrm{O} 2$ & $99.70(6)$ \\
\hline $\mathrm{N} 3-\mathrm{C} 6-\mathrm{C} 9$ & $121.9(2)$ & $\mathrm{N} 5-\mathrm{Cu} 2-\mathrm{Cl} 2$ & $92.21(5)$ \\
\hline $\mathrm{C} 6-\mathrm{C} 7-\mathrm{H} 7$ & 127.0 & $\mathrm{~N} 5-\mathrm{Cu} 2-\mathrm{O} 1$ & $84.77(6)$ \\
\hline $\mathrm{C} 6-\mathrm{C} 7-\mathrm{C} 8$ & 105.95 (19) & $\mathrm{N} 5-\mathrm{Cu} 2-\mathrm{O} 2$ & $81.29(6)$ \\
\hline $\mathrm{C} 8-\mathrm{C} 7-\mathrm{H} 7$ & 127.0 & $\mathrm{O} 1-\mathrm{Cu} 2-\mathrm{Cl} 2$ & $142.41(4)$ \\
\hline $\mathrm{C} 7-\mathrm{C} 8-\mathrm{C} 10$ & $130.0(2)$ & $\mathrm{O} 1-\mathrm{Cu} 2-\mathrm{O} 2$ & $112.12(5)$ \\
\hline $\mathrm{N} 4-\mathrm{C} 8-\mathrm{C} 7$ & 108.08 (19) & $\mathrm{O} 2-\mathrm{Cu} 2-\mathrm{Cl} 2$ & $104.35(4)$ \\
\hline $\mathrm{N} 4-\mathrm{C} 8-\mathrm{C} 10$ & 121.88 (19) & $\mathrm{C} 1-\mathrm{N} 1-\mathrm{H} 1$ & $127.8(15)$ \\
\hline
\end{tabular}




\begin{tabular}{|c|c|c|c|}
\hline C6- C9- $\mathrm{H} 9 \mathrm{~A}$ & 109.5 & $\mathrm{C} 1-\mathrm{N} 1-\mathrm{N} 2$ & $111.74(17)$ \\
\hline $\mathrm{C} 6-\mathrm{C} 9-\mathrm{H} 9 \mathrm{~B}$ & 109.5 & $\mathrm{~N} 2-\mathrm{N} 1-\mathrm{H} 1$ & $120.4(15)$ \\
\hline $\mathrm{C} 6-\mathrm{C} 9-\mathrm{H} 9 \mathrm{C}$ & 109.5 & $\mathrm{C} 3-\mathrm{N} 2-\mathrm{Cu} 1$ & $129.29(15)$ \\
\hline $\mathrm{H} 9 \mathrm{~A}-\mathrm{C} 9-\mathrm{H} 9 \mathrm{~B}$ & 109.5 & $\mathrm{C} 3-\mathrm{N} 2-\mathrm{N} 1$ & $105.97(16)$ \\
\hline $\mathrm{H} 9 \mathrm{~A}-\mathrm{C} 9-\mathrm{H} 9 \mathrm{C}$ & 109.5 & $\mathrm{~N} 1-\mathrm{N} 2-\mathrm{Cu} 1$ & $124.74(13)$ \\
\hline $\mathrm{H} 9 \mathrm{~B}-\mathrm{C} 9-\mathrm{H} 9 \mathrm{C}$ & 109.5 & $\mathrm{C} 6-\mathrm{N} 3-\mathrm{Cu} 1$ & $132.39(15)$ \\
\hline $\mathrm{C} 8-\mathrm{C} 10-\mathrm{H} 10 \mathrm{~A}$ & 109.5 & $\mathrm{C} 6-\mathrm{N} 3-\mathrm{N} 4$ & $107.94(17)$ \\
\hline $\mathrm{C} 8-\mathrm{C} 10-\mathrm{H} 10 \mathrm{~B}$ & 109.5 & $\mathrm{~N} 4-\mathrm{N} 3-\mathrm{Cu} 1$ & $119.66(13)$ \\
\hline $\mathrm{C} 8-\mathrm{C} 10-\mathrm{H} 10 \mathrm{C}$ & 109.5 & $\mathrm{C} 8-\mathrm{N} 4-\mathrm{Cu} 2$ & $132.78(14)$ \\
\hline $\mathrm{H} 10 \mathrm{~A}-\mathrm{C} 10-\mathrm{H} 10 \mathrm{~B}$ & 109.5 & $\mathrm{C} 8-\mathrm{N} 4-\mathrm{N} 3$ & $109.16(16)$ \\
\hline $\mathrm{H} 10 \mathrm{~A}-\mathrm{C} 10-\mathrm{H} 10 \mathrm{C}$ & 109.5 & $\mathrm{~N} 3-\mathrm{N} 4-\mathrm{Cu} 2$ & $117.88(13)$ \\
\hline $\mathrm{H} 10 \mathrm{~B}-\mathrm{C} 10-\mathrm{H} 10 \mathrm{C}$ & 109.5 & $\mathrm{C} 12-\mathrm{N} 5-\mathrm{C} 13$ & $115.41(16)$ \\
\hline $\mathrm{H} 11 \mathrm{~A}-\mathrm{C} 11-\mathrm{H} 11 \mathrm{~B}$ & 108.3 & $\mathrm{C} 12-\mathrm{N} 5-\mathrm{Cu} 2$ & $105.06(12)$ \\
\hline $\mathrm{C} 12-\mathrm{C} 11-\mathrm{H} 11 \mathrm{~A}$ & 109.9 & $\mathrm{C} 12-\mathrm{N} 5-\mathrm{H} 5$ & $111.3(17)$ \\
\hline $\mathrm{C} 12-\mathrm{C} 11-\mathrm{H} 11 \mathrm{~B}$ & 109.9 & $\mathrm{C} 13-\mathrm{N} 5-\mathrm{Cu} 2$ & $111.35(13)$ \\
\hline $\mathrm{O} 1-\mathrm{C} 11-\mathrm{H} 11 \mathrm{~A}$ & 109.9 & $\mathrm{C} 13-\mathrm{N} 5-\mathrm{H} 5$ & $106.2(16)$ \\
\hline $\mathrm{O} 1-\mathrm{C} 11-\mathrm{H} 11 \mathrm{~B}$ & 109.9 & $\mathrm{Cu} 2-\mathrm{N} 5-\mathrm{H} 5$ & $107.3(16)$ \\
\hline $\mathrm{O} 1-\mathrm{C} 11-\mathrm{C} 12$ & $108.86(16)$ & $\mathrm{C} 11-\mathrm{O} 1-\mathrm{Cu} 1$ & $122.25(12)$ \\
\hline $\mathrm{C} 11-\mathrm{C} 12-\mathrm{H} 12 \mathrm{~A}$ & 109.9 & $\mathrm{C} 11-\mathrm{O} 1-\mathrm{Cu} 2$ & $111.54(11)$ \\
\hline $\mathrm{C} 11-\mathrm{C} 12-\mathrm{H} 12 \mathrm{~B}$ & 109.9 & $\mathrm{Cu} 1-\mathrm{O} 1-\mathrm{Cu} 2$ & $110.88(6)$ \\
\hline $\mathrm{H} 12 \mathrm{~A}-\mathrm{C} 12-\mathrm{H} 12 \mathrm{~B}$ & 108.3 & $\mathrm{C} 14-\mathrm{O} 2-\mathrm{Cu} 2$ & $104.53(12)$ \\
\hline $\mathrm{N} 5-\mathrm{C} 12-\mathrm{C} 11$ & $108.97(17)$ & $\mathrm{C} 14-\mathrm{O} 2-\mathrm{H} 2 \mathrm{~A}$ & $108.4(13)$ \\
\hline $\mathrm{N} 5-\mathrm{C} 12-\mathrm{H} 12 \mathrm{~A}$ & 109.9 & $\mathrm{Cu} 2-\mathrm{O} 2-\mathrm{H} 2 \mathrm{~A}$ & $131.5(13)$ \\
\hline $\mathrm{C} 1-\mathrm{C} 2-\mathrm{C} 3-\mathrm{C} 5$ & $-177.8(3)$ & $\mathrm{C} 9-\mathrm{C} 6-\mathrm{C} 7-\mathrm{C} 8$ & $179.7(2)$ \\
\hline $\mathrm{C} 1-\mathrm{C} 2-\mathrm{C} 3-\mathrm{N} 2$ & $0.1(3)$ & $\mathrm{C} 9-\mathrm{C} 6-\mathrm{N} 3-\mathrm{Cu} 1$ & $-0.9(3)$ \\
\hline $\mathrm{C} 1-\mathrm{N} 1-\mathrm{N} 2-\mathrm{C} 3$ & $0.9(2)$ & $\mathrm{C} 9-\mathrm{C} 6-\mathrm{N} 3-\mathrm{N} 4$ & $179.7(2)$ \\
\hline $\mathrm{C} 1-\mathrm{N} 1-\mathrm{N} 2-\mathrm{Cu} 1$ & $-178.30(15)$ & $\mathrm{C} 10-\mathrm{C} 8-\mathrm{N} 4-\mathrm{Cu} 2$ & $2.8(3)$ \\
\hline $\mathrm{C} 2-\mathrm{C} 1-\mathrm{N} 1-\mathrm{N} 2$ & $-0.8(3)$ & $\mathrm{C} 10-\mathrm{C} 8-\mathrm{N} 4-\mathrm{N} 3$ & $177.58(18)$ \\
\hline $\mathrm{C} 2-\mathrm{C} 3-\mathrm{N} 2-\mathrm{Cu} 1$ & $178.58(16)$ & $\mathrm{C} 11-\mathrm{C} 12-\mathrm{N} 5-\mathrm{C} 13$ & $-76.2(2)$ \\
\hline $\mathrm{C} 2-\mathrm{C} 3-\mathrm{N} 2-\mathrm{N} 1$ & $-0.5(3)$ & $\mathrm{C} 11-\mathrm{C} 12-\mathrm{N} 5-\mathrm{Cu} 2$ & $46.87(17)$ \\
\hline $\mathrm{C} 4-\mathrm{C} 1-\mathrm{C} 2-\mathrm{C} 3$ & $178.4(2)$ & $\mathrm{C} 12-\mathrm{C} 11-\mathrm{O} 1-\mathrm{Cu} 1$ & $-111.63(16)$ \\
\hline $\mathrm{C} 4-\mathrm{C} 1-\mathrm{N} 1-\mathrm{N} 2$ & $-179.0(2)$ & $\mathrm{C} 12-\mathrm{C} 11-\mathrm{O} 1-\mathrm{Cu} 2$ & $22.96(19)$ \\
\hline $\mathrm{C} 5-\mathrm{C} 3-\mathrm{N} 2-\mathrm{Cu} 1$ & $-3.4(3)$ & $\mathrm{C} 13-\mathrm{C} 14-\mathrm{O} 2-\mathrm{Cu} 2$ & $41.26(17)$ \\
\hline $\mathrm{C} 5-\mathrm{C} 3-\mathrm{N} 2-\mathrm{N} 1$ & $177.5(2)$ & $\mathrm{C} 14-\mathrm{C} 13-\mathrm{N} 5-\mathrm{C} 12$ & $161.80(17)$ \\
\hline $\mathrm{C} 6-\mathrm{C} 7-\mathrm{C} 8-\mathrm{C} 10$ & $-177.5(2)$ & $\mathrm{C} 14-\mathrm{C} 13-\mathrm{N} 5-\mathrm{Cu} 2$ & $42.16(19)$ \\
\hline $\mathrm{C} 6-\mathrm{C} 7-\mathrm{C} 8-\mathrm{N} 4$ & $1.0(2)$ & $\mathrm{Cu} 1-\mathrm{N} 3-\mathrm{N} 4-\mathrm{C} 8$ & $-178.77(13)$ \\
\hline $\mathrm{C} 6-\mathrm{N} 3-\mathrm{N} 4-\mathrm{C} 8$ & $0.7(2)$ & $\mathrm{Cu} 1-\mathrm{N} 3-\mathrm{N} 4-\mathrm{Cu} 2$ & $-3.11(18)$ \\
\hline $\mathrm{C} 6-\mathrm{N} 3-\mathrm{N} 4-\mathrm{Cu} 2$ & $176.41(13)$ & $\mathrm{N} 1-\mathrm{C} 1-\mathrm{C} 2-\mathrm{C} 3$ & $0.5(3)$ \\
\hline $\mathrm{C} 7-\mathrm{C} 6-\mathrm{N} 3-\mathrm{Cu} 1$ & $179.33(14)$ & $\mathrm{N} 3-\mathrm{C} 6-\mathrm{C} 7-\mathrm{C} 8$ & $-0.6(2)$ \\
\hline $\mathrm{C} 7-\mathrm{C} 6-\mathrm{N} 3-\mathrm{N} 4$ & $-0.1(2)$ & $\mathrm{N} 5-\mathrm{C} 13-\mathrm{C} 14-\mathrm{O} 2$ & $-56.9(2)$ \\
\hline $\mathrm{C} 7-\mathrm{C} 8-\mathrm{N} 4-\mathrm{Cu} 2$ & $-175.87(14)$ & $\mathrm{O} 1-\mathrm{C} 11-\mathrm{C} 12-\mathrm{N} 5$ & $-46.9(2)$ \\
\hline $\mathrm{C} 7-\mathrm{C} 8-\mathrm{N} 4-\mathrm{N} 3$ & $-1.1(2)$ & & \\
\hline
\end{tabular}

Hydrogen-bond geometry $\left(\AA,{ }^{\circ}\right)$ for (shi_4306_)

\begin{tabular}{lllll}
\hline$D-\mathrm{H} \cdots A$ & $D-\mathrm{H}$ & $\mathrm{H} \cdots A$ & $D \cdots A$ & $D-\mathrm{H} \cdots A$ \\
\hline $\mathrm{N} 1-\mathrm{H} 1 \cdots \mathrm{Cl} 2^{\mathrm{i}}$ & $0.87(2)$ & $2.33(2)$ & $3.1201(18)$ & $152(2)$ \\
$\mathrm{N} 5-\mathrm{H} 5 \cdots \mathrm{Cl} 1^{\mathrm{ii}}$ & $0.80(2)$ & $2.84(2)$ & $3.5593(18)$ & $150(2)$ \\
$\mathrm{O} 2-\mathrm{H} 2 A \cdots \mathrm{O} 1^{\mathrm{iii}}$ & $0.85(1)$ & $1.88(1)$ & $2.7264(19)$ & $174(2)$ \\
\hline
\end{tabular}


Symmetry codes: (i) $x+1, y, z$; (ii) $x-1, y, z$; (iii) $-x,-y,-z+1$. 